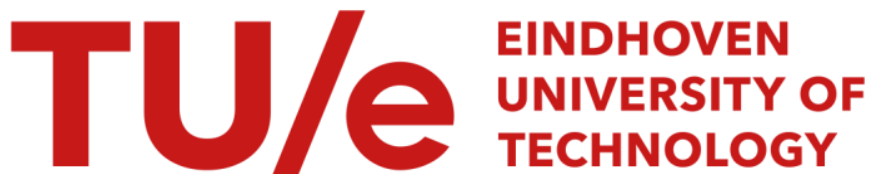

\section{NMR in Magnetite below and around the Verwey transition}

\section{Citation for published version (APA):}

Novák, P., Stepankova, H., Englich, J., Kohout, J., \& Brabers, V. A. M. (2000). NMR in Magnetite below and around the Verwey transition. Physical Review B, 61(2), 1256-1260. https://doi.org/10.1103/PhysRevB.61.1256

DOI:

10.1103/PhysRevB.61.1256

Document status and date:

Published: 01/01/2000

\section{Document Version:}

Publisher's PDF, also known as Version of Record (includes final page, issue and volume numbers)

\section{Please check the document version of this publication:}

- A submitted manuscript is the version of the article upon submission and before peer-review. There can be important differences between the submitted version and the official published version of record. People interested in the research are advised to contact the author for the final version of the publication, or visit the $\mathrm{DOI}$ to the publisher's website.

- The final author version and the galley proof are versions of the publication after peer review.

- The final published version features the final layout of the paper including the volume, issue and page numbers.

Link to publication

\section{General rights}

Copyright and moral rights for the publications made accessible in the public portal are retained by the authors and/or other copyright owners and it is a condition of accessing publications that users recognise and abide by the legal requirements associated with these rights.

- Users may download and print one copy of any publication from the public portal for the purpose of private study or research.

- You may not further distribute the material or use it for any profit-making activity or commercial gain

- You may freely distribute the URL identifying the publication in the public portal.

If the publication is distributed under the terms of Article 25fa of the Dutch Copyright Act, indicated by the "Taverne" license above, please follow below link for the End User Agreement:

www.tue.nl/taverne

Take down policy

If you believe that this document breaches copyright please contact us at:

openaccess@tue.nl

providing details and we will investigate your claim. 


\title{
NMR in magnetite below and around the Verwey transition
}

\author{
P. Novák \\ Institute of Physics, Academy of Sciences of the Czech Republic, Cukrovarnická 10, 16253 Praha 6, Czech Republic \\ H. Štěpánková, J. Englich, and J. Kohout \\ Faculty of Mathematics and Physics, Charles University, V Holešovičkách 2, 18040 Praha 8, Czech Republic \\ V. A. M. Brabers \\ Department of Physics, Eindhoven University of Technology, P.O. Box 513, NL-5600, MB Eindhoven, The Netherlands
}

(Received 29 April 1999)

\begin{abstract}
NMR on ${ }^{57} \mathrm{Fe}$ nuclei was measured in two single crystals of magnetite between 4.2 and $135 \mathrm{~K}$ (temperature of the Verwey transition $T_{V}=121.5$ and $123.9 \mathrm{~K}$ ). We were able to register all lines detected at $4.2 \mathrm{~K}$ also at higher temperatures up to $T_{V}$. The spectra are compatible with the space group $C c$, which predicts 8 and 16 lines for iron on tetrahedral $(A)$ and octahedral $(B)$ sites, respectively. One of the $\operatorname{Fe}(A)$ lines is doubly degenerate for all $T<T_{V}$, which restricts the possible models of the $\mathrm{Fe}^{2+}$ and $\mathrm{Fe}^{3+}$ ordering on the $B$ sites. Above $T_{V}$ no remnant of the low-temperature spectrum was detected. At $4.2 \mathrm{~K}$ the NMR spin-spin and spin-lattice relaxation times were determined.
\end{abstract}

\section{INTRODUCTION}

Magnetite $\mathrm{Fe}_{3} \mathrm{O}_{4}$ is a mixed valence compound with the tetrahedral $(A)$ sublattice completely occupied by ferric ions, while in the octahedral $(B)$ sublattice half of the sites are occupied by $\mathrm{Fe}^{2+}$ and half by $\mathrm{Fe}^{3+}$ ions. At high temperatures the crystal symmetry is cubic and both NMR and Mössbauer spectroscopy see no difference between $\mathrm{Fe}^{2+}$ and $\mathrm{Fe}^{3+}$ ions on the $B$ sites, pointing to the delocalized character of the charge carriers. At the Verwey temperature $T_{V}$ the crystal symmetry is lowered to monoclinic and the electric conductivity drops by more than two orders of magnitude. The NMR spectra of ${ }^{57} \mathrm{Fe}$ on the $B$ sites at $4.2 \mathrm{~K}$ show that the charge carriers are localized or, at least, that their hopping time is much longer comparing to the characteristic time of NMR measurement $\left(10^{-8} \mathrm{~s}\right)$. Despite considerable effort many questions concerning the physics of magnetite below the Verwey transition remain open. In particular there is no consensus concerning the ordering of $\mathrm{Fe}^{2+}$ and $\mathrm{Fe}^{3+}$ on the octahedral sites. By analyzing the angular dependence of NMR spectra Mizoguchi proposed an ordering pattern ${ }^{1}$ which is in conflict with the symmetry group $C c$ found by $\mathrm{x}$-ray, ${ }^{2}$ neutron, ${ }^{3}$ and electron-diffraction ${ }^{4}$ experiments. Moreover, the measurements of the magnetoelectric effect (Ref. 5 and references therein) indicates that at low temperatures the symmetry may be triclinic rather than monoclinic.

$\mathrm{Fe}^{3+}$ ion has half-filled $3 d$ electron shell and zero angular momentum in the ground state. On the other hand, the ground state of the free $\mathrm{Fe}^{2+}$ ion (electron configuration $\left.3 d^{6}\right)$ is ${ }^{5} D$ and considerable orbital momentum remains even when the ${ }^{5} D$ term is split by the crystal field. As a consequence faster NMR relaxation is expected for the ferrous ion, which may help to establish the correspondence between NMR lines and the valency of iron. Yanai et al. ${ }^{9}$ measured the relaxation of several NMR lines, no such analysis was attempted, however.

The dynamics of the charge carriers for temperatures be- low $T_{V}$ is also unclear. From an early study of the nuclear magnetic relaxation in magnetite Mizoguchi and Inoue ${ }^{6} \mathrm{de}-$ duced that above $80 \mathrm{~K}$ the charge carriers move with the frequency higher compared to the NMR frequency. This conclusion was based on an indirect evidence, as they were unable to observe the spectra of iron on the $B$ sites above $14 \mathrm{~K}$ and only the relaxation of $\mathrm{Fe}$ on $A$ sites was studied in the whole temperature range.

The electric conductivity above $T_{V}$ has been explained by Ihle and Lorenz ${ }^{7}$ as a superposition of small polaron band and hopping conductivities. These authors believe that due to a strong intersite Coulomb interaction a considerable shortrange order of $\mathrm{Fe}^{2+}$ and $\mathrm{Fe}^{3+}$ persists above $T_{V}$.

In the present paper the temperature dependence of ${ }^{57} \mathrm{Fe}$ NMR in magnetite is studied by the spin-echo method between 4.2 and $135 \mathrm{~K}$. There exist several earlier measurements of this dependence, ${ }^{8,9}$ but we succeeded in registering all lines detected at the liquid-He temperature also at higher temperatures up to $T_{V}$. At the liquid He temperature the nuclear spin-spin and spin-lattice relaxations were also determined. To ascertain whether a short-range order exists above $T_{V}$ and whether below $T_{V}$ some of the charge carriers give rise to the motionally narrowed spectrum, the NMR close to the Verwey temperature was measured.

\section{EXPERIMENTAL}

In most of the experiments a synthetic $\mathrm{Fe}_{3} \mathrm{O}_{4}$ single crystal with $T_{V}=121.5 \mathrm{~K}$ was used. Measurements at $4.2 \mathrm{~K}$ were repeated using a high-purity synthetic $\mathrm{Fe}_{3} \mathrm{O}_{4}$ single crystal with $T_{V}=123.9 \mathrm{~K}$, for which also NMR spin-lattice relaxation time $T_{1}$ was determined. Very pure crystal is prepared from iron oxide, which is obtained from the decomposition of pure iron oxalate. The crystals were grown by a floatingzone technique using a homebuilt mirror furnace. ${ }^{10}$ NMR spectra were measured by the spin-echo method using the phase-coherent spectrometer with an averaging technique 


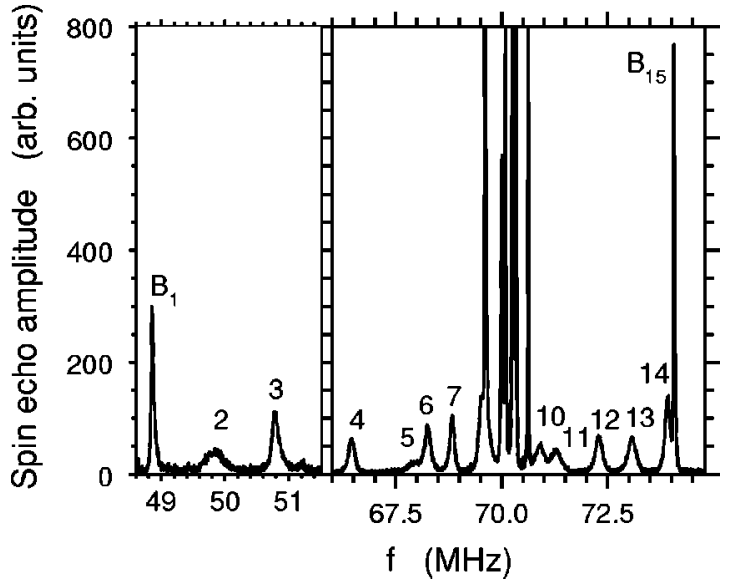

FIG. 1. NMR spectrum of ${ }^{57} \mathrm{Fe}$ in $\mathrm{Fe}_{3} \mathrm{O}_{4}$ single crystal obtained at $4.2 \mathrm{~K}$.

and the complex Fourier transformation. The measurements were made at zero external magnetic field. The high rf power was used in order to excite only the signals from the domains. At $4.2 \mathrm{~K}$ the NMR spectra of both samples were very similar. We did not attempt to anneal the crystals in an external field, where the treatment is known to make the specimen untwinned and leads to narrowing of the NMR lines. ${ }^{1,9}$ The signal-to-noise ratio was significantly improved by using the Carr-Purcel pulse sequence. ${ }^{11}$ Spin-spin relaxation time $T_{2}$ can be then determined from the decrease of the spinecho amplitude in the echo series. This may be performed for every value of the excitation frequency. The spin-lattice relaxation time $T_{1}$ was determined from the dependence of the maximum of the amplitude of the line in question on the repetition time of the two-impulse spin echo. We found, however, that neither spin-spin nor spin-lattice relaxation are strictly exponential.

\section{RESULTS}

The spectrum measured at $4.2 \mathrm{~K}$ is shown in Fig. 1. In Fig. 2 the region of the intensive $\mathrm{Fe}(A)$ lines is presented. The resonance frequencies as well as the lineshapes are very close to the ones observed by Mizoguchi. ${ }^{1}$ The spectrum at $121 \mathrm{~K}$ is shown in Fig. 3, while the region of $\mathrm{Fe}(A)$ lines at $118 \mathrm{~K}$ is displayed in Fig. 4. It is seen that at $4.2 \mathrm{~K}$ the line

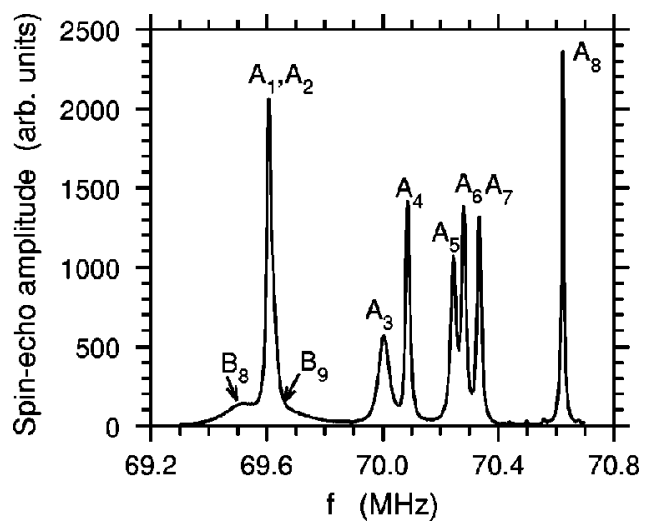

FIG. 2. Region of $\mathrm{Fe}^{3+}(A)$ lines at $4.2 \mathrm{~K}$.

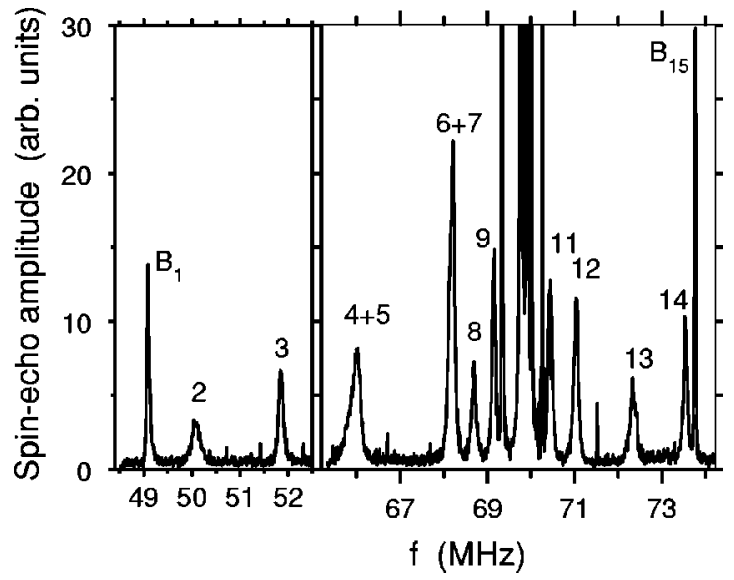

FIG. 3. NMR spectrum of ${ }^{57} \mathrm{Fe}$ obtained at $121 \mathrm{~K}$.

$B 9$, and partially also $B 8$, are masked by intensive lines $A 1$, $A 2$. At elevated temperatures, due to the steeper temperature dependence of the resonance frequency, $B 8$ and $B 9$ lines come out of the $\operatorname{Fe}(A)$ spectrum, while $B 10$ and $B 11$ lines occurs within it. The temperature dependence of the NMR frequencies is shown in Fig. 5. The resonance frequency, linewidth, and relaxation times of the NMR lines at the liquid He temperature are summarized in Table I. In order to compare the intensity of the NMR lines we performed a standard $f^{2}$ correction of the spectrum. Moreover, we also took into account the fact that $T_{2}$ is different for different lines and reconstructed the spectrum in the limit $\tau \rightarrow 0$, where $\tau$ is the time interval between the exciting and refocusing radio frequency pulses. In Table I the areas $\mathcal{S}$ under the lines of such reconstructed spectrum are given. $T_{1}, T_{2}$, and $\mathcal{S}$ for the line $B 9$ could not be reliably determined, hence these values are omitted. Because the $f^{2}$ correction is only a rough approximation to the frequency dependence of the spin-echo amplitude and because the relaxation is not strictly exponential the values of $\mathcal{S}$ should be taken with some caution. Nevertheless, we can conclude with some confidence that the line at $69.606 \mathrm{MHz}$ is a double line. It retains the doubled intensity also at higher temperatures as clearly seen in Fig. 4. With possible exception of the line $A 3$, relative intensities of the NMR lines are similar $(0.79<\mathcal{S}<1.21)$ indicating that the lines originate from the same number of ${ }^{57} \mathrm{Fe}$ nuclei.

Above $T_{V}$ the spectrum is reduced to two lines as ex-

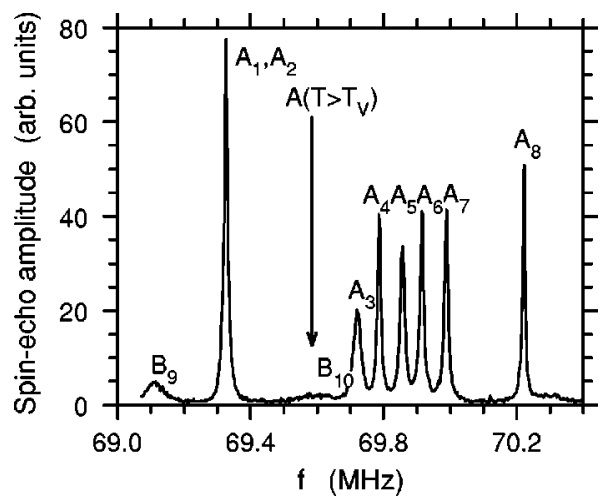

FIG. 4. Region of $\mathrm{Fe}^{3+}(A)$ lines at $118 \mathrm{~K}$. The arrows indicate the frequency of eventual motionally narrowed spectrum. 

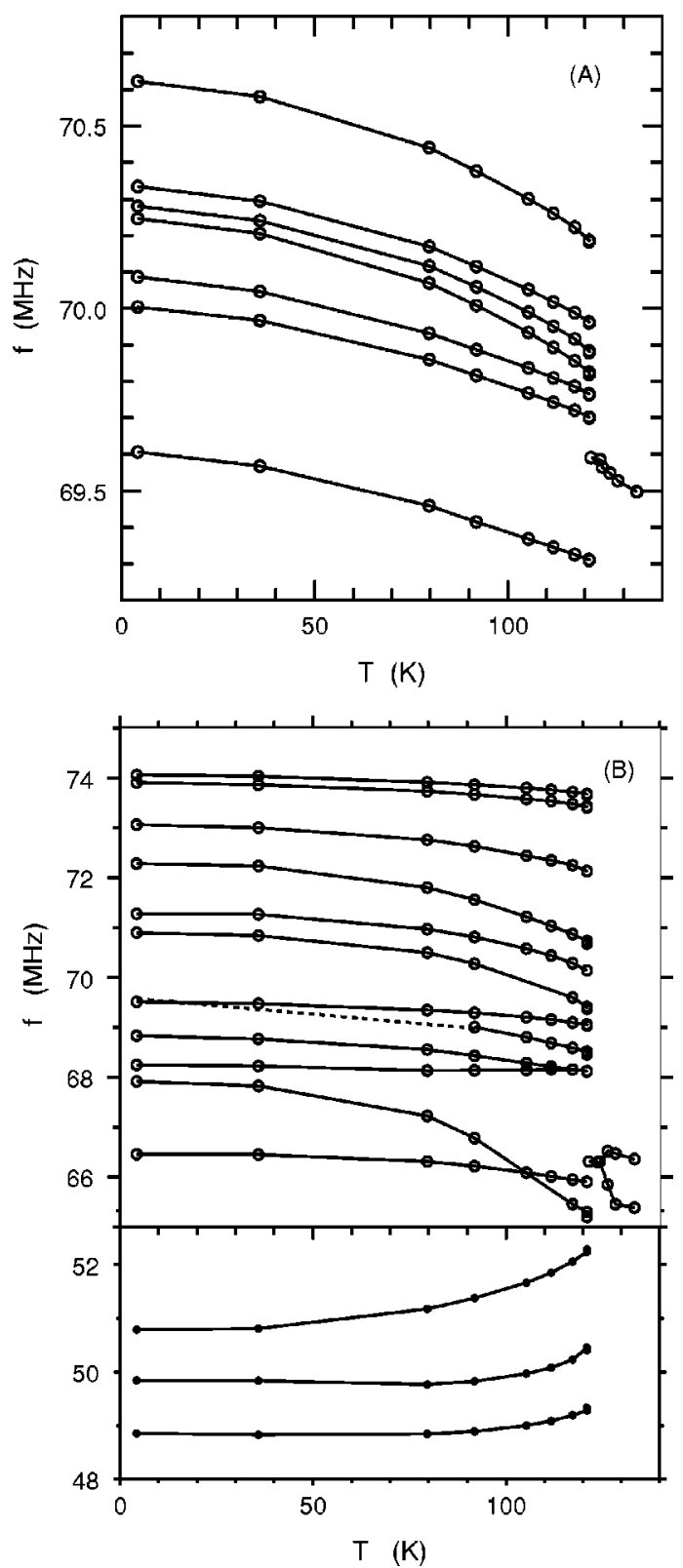

TABLE I. Resonance frequency $f_{\text {res }}$, linewidth $\Delta$, spin-lattice relaxation time $T_{1}$ and spin-spin relaxation time $T_{2}$, and area $S$ of NMR lines in magnetite at $4.2 \mathrm{~K}$.

\begin{tabular}{|c|c|c|c|c|c|}
\hline Line & $f_{\text {res }}(\mathrm{MHz})$ & $\Delta(\mathrm{MHz})$ & $T_{1}(\mathrm{~s})$ & $T_{2}(\mathrm{~ms})$ & $\mathcal{S}$ (arb. units) \\
\hline$B 1$ & 48.856 & 0.044 & 6.6 & 59.2 & 1.19 \\
\hline$B 2$ & 49.839 & 0.340 & 7.1 & 16.2 & 1.16 \\
\hline$B 3$ & 50.783 & 0.125 & 5.4 & 7.4 & 1.05 \\
\hline$B 4$ & 66.459 & 0.178 & 10.6 & 12.3 & 0.94 \\
\hline$B 5$ & 67.900 & 0.310 & 10.9 & 14.9 & 0.90 \\
\hline$B 6$ & 68.249 & 0.188 & 8.5 & 27.8 & 1.09 \\
\hline$B 7$ & 68.840 & 0.124 & 10.3 & 7.3 & 0.83 \\
\hline$B 8$ & 69.507 & 0.086 & 7.4 & 22.5 & 0.95 \\
\hline$B 9$ & 69.630 & $\approx 0.2$ & & & \\
\hline$B 10$ & 70.900 & 0.225 & 8.2 & 7.8 & 0.94 \\
\hline$B 11$ & 71.285 & 0.287 & 9.2 & 9.0 & 0.79 \\
\hline$B 12$ & 72.292 & 0.188 & 8.2 & 11.7 & 0.88 \\
\hline$B 13$ & 73.071 & 0.215 & 8.2 & 16.8 & 0.99 \\
\hline$B 14$ & 73.908 & 0.065 & 7.8 & 97.4 & 1.11 \\
\hline$B 15$ & 74.061 & 0.031 & 6.5 & 220.9 & 1.03 \\
\hline$A 1+A 2$ & 69.606 & 0.019 & 7.9 & 197 & 2.42 \\
\hline$A 3$ & 70.004 & 0.040 & 8.3 & 273 & 1.48 \\
\hline$A 4$ & 70.087 & 0.015 & 7.9 & 240 & 1.20 \\
\hline$A 5$ & 70.245 & 0.028 & 7.8 & 161 & 1.08 \\
\hline$A 6$ & 70.280 & 0.017 & 7.8 & 201 & 1.13 \\
\hline$A 7$ & 70.334 & 0.015 & 7.8 & 239 & 1.17 \\
\hline$A 8$ & 70.622 & 0.009 & 7.6 & 446 & 1.12 \\
\hline
\end{tabular}

above $T_{V}$ is best demonstrated using the intensive and sharp spectra of the $A$ sites. The inspection of Fig. 6 reveals only a single $\mathrm{Fe}(A)$ line and no remnants of the low-temperature NMR spectrum. Consequently no static short-range order is present above $T_{V}$. However, there may be a short-range order which fluctuates with the frequency higher than the NMR frequency.

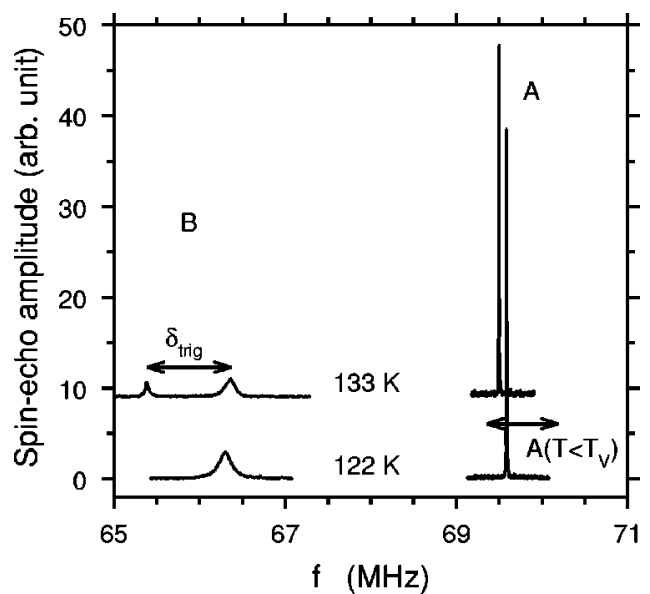

FIG. 6. NMR spectrum of ${ }^{57} \mathrm{Fe}$ in $\mathrm{Fe}_{3} \mathrm{O}_{4}$ single crystal above the Verwey temperature $T_{V}=121.5 \mathrm{~K}$. At $122 \mathrm{~K}$ the magnetization is along $\langle 100\rangle$ and the leftright arrow indicates the region of the $\mathrm{Fe}(A)$ spectrum for $T$ below $T_{V}$. At $133 \mathrm{~K}$ the magnetization is along $\langle 111\rangle$ direction. $\delta_{\text {trig }}$ is the splitting caused by the trigonal anisotropy of the hyperfine field.

First we discuss the spectra in the vicinity of the Verwey transition. The eventual presence of the short-range order

$\langle 100\rangle$ direction. The very narrow line at $69.590 \mathrm{MHz}$ corresponds to $\mathrm{Fe}^{3+}$ ions on the $A$ sites, while $\mathrm{Fe}(B)$ ions give rise to the line at $66.297 \mathrm{MHz}$. At $\approx 125 \mathrm{~K}$ the system undergoes a $\langle 100\rangle \rightarrow\langle 111\rangle$ spin reorientation transition, which leads to the splitting of the $\mathrm{Fe}(B)$ line to two lines with ideal intensity ratio $1: 3$. The line at lower frequency corresponds to the $B$ sites for which the local trigonal axis is parallel to the magnetization, while the remaining $B$ sites give rise to the higher frequency line. The development of the NMR spectrum above $T_{V}$ is shown in Fig. 6.

\section{DISCUSSION}


Within the sensitivity of our measurement the NMR spectrum retains its character in the whole temperature interval $4.2 \mathrm{~K} \leqslant T<T_{V}$, in particular there is no trace of the motionally narrowed $\mathrm{Fe}(A)$ spectrum close to $T_{V}$ (Fig. 4). As the charge carriers are believed to be localized at low temperatures, we conclude that for all temperatures below $T_{V}$ the charge carriers either remain localized, or at least they move with the characteristic time much longer compared to the characteristic time of the NMR measurement (1.5 $\left.\times 10^{-8} \mathrm{~s}\right)$.

The NMR frequency $f$ is connected with the effective magnetic field $\vec{B}$ by

$$
f=\gamma|\vec{B}| / 2 \pi
$$

where $\gamma$ is the gyromagnetic ratio. $\vec{B}$ is the sum

$$
\vec{B}=\hat{A} \vec{\mu}+\vec{B}_{\mathrm{dip}}
$$

$\hat{A}$ and $\vec{\mu}$ are the hyperfine coupling tensor and the electronic magnetic moment, respectively. The temperature dependence of $f$ below the Verwey transition is connected with the change of both $\hat{A}$ and $\vec{\mu}$. While $|\vec{\mu}|$ decreases monotonously with the increasing temperature, $\hat{A}(T)$ reflects the temperature dependence of the ordering parameter $p$ and for inequivalent sites it may have different character. Above $T_{V}$ $p=0$ and there is only one $\operatorname{Fe}(B)$ line at $f_{B}\left(T_{V}\right)=66.297$ $\mathrm{MHz}$. It is therefore not surprising that for $\mathrm{Fe}(B)$ lines with $f<53 \mathrm{MHz} \hat{A}(T)$ dependence dominates and the frequency of these lines increases with increasing temperature. $f(T)$ of the line $B 5$ (Fig. 5) is of interest. For $T \geqslant 100 \mathrm{~K}$ its frequency is lower than $f_{B}\left(T_{V}\right)$, while it is higher at low temperatures. This behavior may be understood if we take into account that there are several different ordering parameters, one concerns the crystal structure, the other corresponds to the ordering of iron valencies (this is an oversimplification as more ordering parameters are involved ${ }^{12}$ ). NMR frequencies observed below $T_{V}$ reflect only the change of the crystal structure ${ }^{13}$ and $\mu$ and they are not influenced by eventual decrease of the valency ordering. The static change of the valency ordering would result in broadening of the lines, while the dynamic change connected with the hopping would produce a distinct, motionally narrowed spectrum, which is not observed.

We now turn to the problem of the crystal symmetry and ordering of iron valencies below $T_{V}$. The monoclinic cell of $\mathrm{Fe}_{3} \mathrm{O}_{4}$ contains 32 formula units with $32 A$ and $64 B$ sites, which numbers are reduced by $C c$ symmetry to $8 A$ and $16 B$ crystallographically inequivalent sites. For magnetization along [001] (easy direction of magnetization at low temperatures) the presence of magnetization does not reduce the symmetry, so there are 8 and 16 magnetically inequivalent iron ions on $A$ and $B$ sites, respectively. As a consequence, using the symmetry only, $16 \mathrm{Fe}(B)$ and $8 \mathrm{Fe}(A)$ lines are predicted in the NMR spectrum below $T_{V}$. Indeed we observed eight lines corresponding to $\mathrm{Fe}^{3+}$ ions on the $A$ sites, but one of the $\operatorname{Fe}(B)$ lines is missing. It might be, however, hidden in the intensive $\operatorname{Fe}(A)$ spectrum (cf. rather large intensity of line $A 3$ in Table I). Though further effort to find the missing $B$ line is desirable, we can conclude that the NMR spectra observed below $T_{V}$ are in accord with the $C c$ symmetry of magnetite. In this context the fact that we have observed the $B 9$ line is important. Note that its existence could only be ascertained by careful measurement of the temperature dependence-this is the reason why only $14 \mathrm{~B}$ lines were found earlier. ${ }^{1}$

As half of the $B$ sites is occupied by $3+$ and other half by $2+$ iron, eight of the $\mathrm{Fe}(B)$ lines should correspond to $\mathrm{Fe}^{3+}$ and eight to $\mathrm{Fe}^{2+}$ ions. This contradicts Mizoguchi, ${ }^{1}$ who ascribed only five of the $B$ lines to the ferric ions, consequently his ordering scheme of iron valencies cannot be reconciled with the $C c$ symmetry. The main basis of the Mizoguchi ordering scheme comes from the measurement of the angular dependence of the five NMR lines ascribed to the $\mathrm{Fe}^{3+}(B)$ ions. The magnitude of corresponding anisotropy of the resonance frequencies is larger than $2.5 \mathrm{MHz}$, at least for some of the lines. Above $T_{V}$ we found that the anisotropy caused by the trigonal local distortion of the octahedral sites is $1.2 \mathrm{MHz}$ (it is equal to $9 / 8$ of the splitting $\delta_{\text {trig }}$ between $\mathrm{Fe}(B)$ lines in Fig. 6). Below $T_{V}$ the magnetic moment increases by $\approx 1 \%$ only and the increase of the trigonal anisotropy by more than a few percent would be surprising. The anisotropy connected with the trigonal distortion is thus only smaller part of the anisotropy found in Ref. 1. This contradicts one of the assumptions made by Mizoguchi when analyzing the angular dependence, explaining therefore why his proposal for the ordering may be incorrect.

A conspicuous feature of the spectra observed is the doubling of the lowest frequency $\operatorname{Fe}(A)$ line. As this line is not broadened when the temperature is increased, we can conclude with some confidence that two formally inequivalent $\mathrm{Fe}^{3+}$ ions, which give rise to this line, have nearly the same oxygen environment and also their effective exchange fields $H_{\text {exch }}$ are equal. Analysis of the line shape shows that the difference of $H_{\text {exch }}$ larger than $2 \%$ would already lead to detectable splitting of the line. Equality of $H_{\text {exch }}$ means that also the cation environment of the two sites in question must be very similar, requiring the same number of nearest $\mathrm{Fe}^{3+}(B)$ and $\mathrm{Fe}^{2+}(B)$ neighbors and very close geometry of the $\mathrm{Fe}(B)-\mathrm{O}^{2-}-\mathrm{Fe}^{3+}(A)$ triads. This provides a stringent condition for the arrangement of iron valencies on the $B$ sublattice. Until now all models of this arrangement make use of the Anderson condition for the short-range ordering, ${ }^{14}$ which states that in every tetrahedron formed by nearestneighbor $B$ sites there are two $\mathrm{Fe}^{3+}$ and two $\mathrm{Fe}^{2+}$ ions. As showed by Zuo et ll $^{4}$ there are only nine models (leaving out the original proposal of Verwey) which possess the $C c$ symmetry and comply with the Anderson condition. We made an analysis of the geometry of $\mathrm{Fe}^{3+}(A)$ environment using the positional parameters given by Iizumi et $a .^{3}$ Only one of the nine models-model 6 in the notation of Ref. 4 - satisfies the requirement that relative positions of the oxygen ligands and the nearest $B$ neighbors of two of $\mathrm{Fe}^{3+}(A)$ ions is the same (model 2 is preferred by Zuo et $a l .,{ }^{4}$ on the basis of the electron-diffraction study). We have to note, however, that unfortunately in order to facilitate the structural analysis Iizumi et ll $^{3}$ assumed orthorhombic $P m c 2_{1}$ symmetry group and it is difficult to estimate the effect of this approximation on the calculation of the exact geometry. 
Inspection of Table I reveals that the spin-lattice relaxation time $T_{1}$ is almost the same for all resonance lines and, with the exception of three lines, also $T_{2}$ of the $B$ lines has the same order of magnitude. This does not correspond to the usual situation, when $\mathrm{Fe}^{2+}$ relaxation is much faster, and it indicates that there may be a strong mixing of $3 d^{5}$ electron configuration of $\mathrm{Fe}^{3+}(B)$ with the $3 d^{6}$ configuration of $\mathrm{Fe}^{2+}(B)$. Such strong mixing is further supported by a bondlength analysis, which we performed using the structure data of Ref. 3. For the nine models of the valency ordering, which satisfy both the Anderson condition and the $C c$ symmetry, the ionic radii of $\mathrm{Fe}^{2+}(B)$ and $\mathrm{Fe}^{3+}(B)$ were calculated. The result is surprising - within all models the difference of these radii $\Delta R_{\text {ion }}$ is very small (maximal $\Delta R_{\text {ion }}=0.01 \AA$ ), contrasting with $\Delta R_{\text {ion }}=0.135 \AA$ calculated using the conventional ionic radii of $\mathrm{Fe}^{2+}(B)$ and $\mathrm{Fe}^{3+}(B)$ as given in Ref. 15.

\section{CONCLUSIONS}

The analysis of the NMR spectra below and around the Verwey transition brought several significant results. Above $T_{V}$ the spectra reveal that if the short-range order exists for $T>T_{V}$ it must fluctuate rapidly. Below $T_{V}$ we showed that the NMR spectra are in accord with the $C c$ symmetry and the reason was given why the earlier analysis ${ }^{1}$ of these spectra may be incorrect. Finally, and perhaps most importantly, the NMR relaxation results and also the bond length analysis indicate that below $T_{V}$ the states of iron ions on the $B$ sublattice are mixed so strongly that the notion of $2+$ and $3+$ valency may lose its meaning.

\section{ACKNOWLEDGMENTS}

This work was supported by Grants No. 202/97/1018 and 202/97/P070 of the Grant Agency of the Czech Republic and by Grant No. GAUK 25/97/B-FYZ/MFF.
${ }^{1}$ M. Mizoguchi, J. Phys. Soc. Jpn. 44, 1501 (1978); 44, 1512 (1978).

${ }^{2}$ J. Yoshida and S. Iida, J. Phys. Soc. Jpn. 42, 230 (1977).

${ }^{3}$ M. Iizumi, T. F. Koetzle, G. Shirane, S. Chikazumi, M. Matsui, and S. Todo, Acta Crystallogr., Sect. B: Struct. Crystallogr. Cryst. Chem. 38, 2121 (1982).

${ }^{4}$ J. M. Zuo, J. C. H. Spence, and W. Petuskey, Phys. Rev. B 42, 8451 (1990).

${ }^{5}$ Y. Myiamoto and M. Shindo, J. Phys. Soc. Jpn. 62, 1423 (1993).

${ }^{6}$ T. Mizoguchi and M. Inoue, J. Phys. Soc. Jpn. 21, 1310 (1966).

${ }^{7}$ D. Ihle and B. Lorenz, J. Phys. C 18, L647 (1985).

${ }^{8}$ N. M. Kovtun and A. A. Shemyakov, Solid State Commun. 13,
1345 (1973).

${ }^{9}$ K. Yanai, M. Mizoguchi, and S. Iida, J. Phys. Soc. Jpn. 50, 65 (1981).

${ }^{10}$ A. J. M. Kuipers and V. A. M. Brabers, Phys. Rev. B 14, 1401 (1976).

${ }^{11}$ C. P. Slichter, Principles of Magnetic Resonance, Springer Series in Solid-State Sciences (Springer-Verlag, Berlin, 1978).

${ }^{12}$ J. R. Cullen and E. R. Callen, Phys. Rev. B 7, 397 (1973).

${ }^{13}$ J. Yoshida and S. Iida, J. Phys. Soc. Jpn. 47, 1627 (1979).

${ }^{14}$ P. W. Anderson, Phys. Rev. 102, 1008 (1956).

${ }^{15}$ R. D. Shannon, Acta Crystallogr., Sect. A: Cryst. Phys., Diffr., Theor. Gen. Crystallogr. 32, 751 (1976). 